\title{
Synchronization of stochastic multiple weighted coupled networks with Markovian switching
}

\author{
Xupan Yao ${ }^{1}$, Chunmei Zhang ${ }^{1 *}$ and Dan Xia ${ }^{1}$
}

\author{
"Correspondence: \\ Zhangcm87@home.swjtu.edu.cn \\ 'School of Mathematics, Southwest \\ Jiaotong University, Chengdu, China
}

\begin{abstract}
We investigate the synchronization of stochastic multiple weighted coupled networks with Markovian switching (SMWCNMS). By designing an appropriate controller, we obtain several sufficient criteria ensuring the $p$ th moment exponential synchronization and almost surely exponential synchronization for SMWCNMS based on graph theory. Moreover, we also investigate the pth moment asymptotical synchronization and almost surely asymptotical synchronization for SMWCNMS. Finally, we provide a numerical example to illustrate the availability of the proposed synchronization criteria.
\end{abstract}

Keywords: Stochastic coupled networks; Multi-weights; Exponential synchronization; Asymptotical synchronization

\section{Introduction}

During the past decades, coupled networks have attracted attention of many mathematicians and other scientists. They have extensive applications in various fields, such as power engineering and secure communication [1-6]. Therein, synchronization, as one of the most important dynamic characteristics of coupled networks, plays a significant role in many fields such as biological systems, chemical reactions, and information technology [7-14]. As is well known, when coupled networks are not synchronized by themselves, some controllers are always designed to ensure the synchronization of coupled networks. As an effective technique, the feedback control has drawn great attention from researchers [15-18].

In real applications, systems are always perturbed by uncertain disturbances [19-21]. In the coupled networks, the inevitable uncertainty should be considered. One is white noise, and the other is color noise. Researchers always use stochastic coupled systems driven by Brownian motion to describe complex networks with white noise [18, 22]. Telegraph noise, which is a simple color noise, can be described as a switching between two or more environmental nodes. Markov chains can describe a system in which state changes jumping from one state to another at different times. Therefore coupled networks with Markovian switching can better describe telegraph noise, whereas deterministic systems or stochastic

(c) The Author(s) 2020. This article is licensed under a Creative Commons Attribution 4.0 International License, which permits use, sharing, adaptation, distribution and reproduction in any medium or format, as long as you give appropriate credit to the original author(s) and the source, provide a link to the Creative Commons licence, and indicate if changes were made. The images or other third party material in this article are included in the article's Creative Commons licence, unless indicated otherwise in a credit line to the material. If material is not included in the article's Creative Commons licence and your intended use is not permitted by statutory regulation or exceeds the permitted use, you will need to obtain permission directly from the copyright holder. To view a copy of this licence, visit http://creativecommons.org/licenses/by/4.0/. 
systems driven by Brownian motion mentioned above cannot explain it. Synchronization of coupled networks with Markovian switching has received extensive attention [23-27].

Many real-world networks, such as social, transportation, and biology networks, can be coupled by multiple coupling forms. To describe these networks more accurately, multiweighted coupled network models should be established. Some researchers also call them as multilink networks. In the multi-weighted networks, different weights have different properties. Hence they cannot simply merge into a single-weighted network [28, 29]. For instance, in the social network, people can communicate with others by multiple ways, such as mobile phone, Wechat, E-mail, and so on. Since every contact way has different influence, social network can be described as a multi-weighted coupled network in a more accurate sense. Therefore it is necessary and interesting to investigate the synchronization of multi-weighted coupled networks [22,30-33]. This investigation has wide applications in many fields such as public traffic network and Chua's circuits [32, 34, 35]. For example, in [32] the authors modeled public traffic network as a complex network with triplet weights, that is, the departing frequency, the passenger flow density, and the coefficient of bus line length. Therein they showed the impact of triplet weights on the balance of whole complex network.

In the literature, results are mainly about the synchronization of stochastic singleweighted coupled networks with Markovian switching or determined multi-weighted coupled networks. There exist few related results about the synchronization of stochastic multi-weighted coupled networks with Markovian switching (SMWCNMS). Hence it is essential for us to accomplish this task in this paper. In the literature, various types of methods have been consecutively emerged to enrich the research topic of synchronization. For instance, by using the Kronecker product method, Qiu et al. addressed the synchronization problem for a class of complex dynamical networks with and without coupling delay [36]. Other synchronization techniques have also imported, such as the linear matrix inequality method, M-matrix approach, and so on.

In this paper, the influence of both multiple weights and Markovian switching is considered in the coupled networks. This makes it difficult to study the synchronization of SMWCNMS by the method mentioned. We need to try some new method. Li and Shuai [37] proposed a systematic approach to construct global Lyapunov functions for largescale coupled systems from building blocks of individual vertex systems with the help of some results in graph theory. This graph-theoretic method can avoid the difficulty of directly constructing the global Lyapunov function of coupled networks and has been considered as an important method for dynamic behavior analysis of coupled networks. Inspired by this method, some relevant results have been published in [22, 26, 38-42]. In [26], by using this graph-theoretical technique, some novel sufficient criteria are derived to ensure the exponential synchronization for stochastic coupled networks with Markovian switching. In [22], exponential synchronization of stochastic complex networks with multi-weights has also been obtained by the graph-theoretic approach.

Inspired by these discussions, this paper tends to investigate the synchronization of SMWCNMS with the help of graph-theoretic approach and stochastic analysis theory. Compared with results in the literature, the contribution of the paper is threefold. Firstly, stochastic coupled systems with Markovian switching on multiple subnetworks are utilized to model stochastic complex networks with multi-weights and telegraph noise. Then, based on the drive-response concept, we combine a novel graph-theoretic method and 
M-matrix theory to investigate the synchronization of drive-response networks. We provide both exponential synchronization criteria and asymptotical synchronization criteria. Finally, we give numerical simulations to show the applicability and effectiveness of theoretical results.

This paper is arranged as follows. Firstly, we recall some results in graph theory and give model description in Sect. 2. Secondly, in Sect. 3, we present exponential synchronization and asymptotical synchronization of SMWCNMS. To illustrate the theoretical results, in Sect. 4, we give a numerical example. Finally, we close this paper with concluding remarks in Sect. 5.

\section{Preliminary and model description}

First, we give some useful notations. Let $(\Omega, \mathcal{F}, \mathbb{F}, \mathbb{P})$ be a complete probability space with a filtration $\mathbb{F}=\left\{\mathcal{F}_{t}\right\}_{t \geq 0}$ satisfying the usual conditions (the filtration is right-continuous, and $\mathcal{F}_{0}$ contains all $\mathbb{P}$-null sets); $W$ is a scalar standard Brownian motion defined on the given probability space, and $\mathbb{E}$ denotes the mathematical expectation with respect to $\mathbb{P}$. Let $|\cdot|$ be the Euclidean norm for vectors or the trace norm for matrices, and let $\mathbb{R}_{+}^{n}=\left\{x \in \mathbb{R}^{n}: x_{i}>0, i=1,2, \ldots, n\right\}$. We denote by $C^{2,1}\left(\mathbb{R}^{n} \times \mathbb{R}_{+}^{1} ; \mathbb{R}_{+}^{1}\right)$ the family of all nonnegative function $V(x, t)$ on $\mathbb{R}^{n} \times \mathbb{R}_{+}^{1}$ that are twice continuously differentiable in $x$ and once in $t$. We denote by $L^{p}\left(\mathbb{R}_{+}^{1} ; \mathbb{R}_{+}^{1}\right)$ the family of positive random variables $y$ such that $\mathbb{E}|y|^{p}<\infty$. Moreover, $a \vee b \triangleq \max \{a, b\}$ and $a \wedge b \triangleq \min \{a, b\}$. Let $r$ be a right-continuous Markov chain on $(\Omega, \mathcal{F}, \mathbb{F}, \mathbb{P})$ taking values in a finite state space $\mathbb{S}=\{1,2, \ldots, m\}$. The corresponding generator $\Gamma=\left(\gamma_{i j}\right)_{m \times m}$ is given by

$$
\mathbb{P}\{r(t+\Delta)=j \mid r(t)=i\}= \begin{cases}\gamma_{i j} \Delta+o(\Delta), & i \neq j, \\ 1+\gamma_{i j} \Delta+o(\Delta), & i=j .\end{cases}
$$

Let $\mathcal{G}$ be a weighted digraph with $N$ vertices and $l$ different kinds of weights. By the network split method [32, 43], coupled networks with multi-weights can be split into $l$ subnetworks. Then coupled networks with multi-weights and Markovian switching on $\mathcal{G}$ can be described as

$$
\begin{aligned}
\dot{x}_{k}(t)= & f_{k}\left(x_{k}(t), t, r(t)\right)+\delta_{1}(r(t)) \sum_{h=1}^{N} b_{k h}^{1}(r(t)) \bar{H}_{1}\left(x_{h}(t), t, r(t)\right) \\
& +\delta_{2}(r(t)) \sum_{h=1}^{N} b_{k h}^{2}(r(t)) \bar{H}_{2}\left(x_{h}(t), t, r(t)\right)+\cdots \\
& +\delta_{l}(r(t)) \sum_{h=1}^{N} b_{k h}^{l}(r(t)) \bar{H}_{l}\left(x_{h}(t), t, r(t)\right), \quad k=1,2, \ldots, N
\end{aligned}
$$

where $x_{k} \in \mathbb{R}^{n}$ is the state variable. The meanings of other parameters are shown as follows:

$f_{k}: \mathbb{R}^{n} \times \mathbb{R}_{+}^{1} \times \mathbb{S} \rightarrow \mathbb{R}^{n}$ is continuously differentiable;

$\delta_{s}(r(t))(s=1,2, \ldots, l)$ is the coupling strength of the $s$ th subnetwork;

$\bar{H}_{s}: \mathbb{R}^{n} \times \mathbb{R}_{+}^{1} \times \mathbb{S} \rightarrow \mathbb{R}^{n}(s=1,2, \ldots, l)$ shows the inner coupling;

$b_{k h}^{s}(s=1,2, \ldots, l)$ is the $s$ th weight from the $h$ th node to the $k$ th node if it exists and 0 otherwise. 
We treat network (1) as a drive system. Then, to synchronize this system via feedback control, we can design the following response system with white noise and Markovian switching:

$$
\begin{aligned}
\mathrm{d} y_{k}(t)= & {\left[f_{k}\left(y_{k}(t), t, r(t)\right)+\delta_{1}(r(t)) \sum_{h=1}^{N} b_{k h}^{1}(r(t)) \bar{H}_{1}\left(y_{h}(t), t, r(t)\right)\right.} \\
& +\delta_{2}(r(t)) \sum_{h=1}^{N} b_{k h}^{2}(r(t)) \bar{H}_{2}\left(y_{h}(t), t, r(t)\right)+\cdots \\
& \left.+\delta_{l}(r(t)) \sum_{h=1}^{N} b_{k h}^{l}(r(t)) \bar{H}_{l}\left(y_{h}(t), t, r(t)\right)+u_{k}(t, r(t))\right] \mathrm{d} t \\
& +g_{k}\left(y_{k}(t)-x_{k}(t), t, r(t)\right) \mathrm{d} W(t), \quad k=1,2, \ldots, N,
\end{aligned}
$$

where $y_{k} \in \mathbb{R}^{n}$ is the state variable of response system, and $g_{k}: \mathbb{R}^{n} \times \mathbb{R}_{+}^{1} \times \mathbb{S} \rightarrow \mathbb{R}^{n}$ is the perturbation intensity of white noise on the $k$ th vertex system.

For any given initial data $x_{0}, y_{0} \in \mathbb{R}^{n N}$ and $r_{0} \in \mathbb{S}$, the existence of unique solution to the drive-response systems (1) and (2) can be guaranteed under some conditions. For example, the coefficients of drive system (1) and response system (2) satisfy the linear growth condition and the local Lipschitz condition [44]. Let $e(t)=y(t)-x(t)=$ $\left(e_{1}^{\mathrm{T}}(t), e_{2}^{\mathrm{T}}(t), \ldots, e_{N}^{\mathrm{T}}(t)\right)^{\mathrm{T}}$ be the synchronization error, where $x(t)=\left(x_{1}^{\mathrm{T}}(t), x_{2}^{\mathrm{T}}(t), \ldots, x_{N}^{\mathrm{T}}(t)\right)^{\mathrm{T}}$, $y(t)=\left(y_{1}^{\mathrm{T}}(t), y_{2}^{\mathrm{T}}(t), \ldots, y_{N}^{\mathrm{T}}(t)\right)^{\mathrm{T}}, e_{k}=y_{k}-x_{k} \triangleq\left(e_{k}^{(1)}, e_{k}^{(2)}, \ldots, e_{k}^{(n)}\right)^{\mathrm{T}}, k=1,2, \ldots, N$. We also denote $F_{k}\left(e_{k}(t), t, r(t)\right)=f_{k}\left(y_{k}(t), t, r(t)\right)-f_{k}\left(x_{k}(t), t, r(t)\right)$ and $H_{s}\left(e_{h}(t), t, r(t)\right)=$ $\bar{H}_{s}\left(y_{h}(t), t, r(t)\right)-\bar{H}_{s}\left(x_{h}(t), t, r(t)\right)$. The dynamical system of synchronization error between coupled networks (1) and (2) can be described as

$$
\begin{aligned}
\mathrm{d} e_{k}(t)= & {\left[F_{k}\left(e_{k}(t), t, r(t)\right)+\delta_{1}(r(t)) \sum_{h=1}^{N} b_{k h}^{1}(r(t)) H_{1}\left(e_{h}(t), t, r(t)\right)\right.} \\
& +\delta_{2}(r(t)) \sum_{h=1}^{N} b_{k h}^{2}(r(t)) H_{2}\left(e_{h}(t), t, r(t)\right)+\cdots \\
& \left.+\delta_{l}(r(t)) \sum_{h=1}^{N} b_{k h}^{l}(r(t)) H_{l}\left(e_{h}(t), t, r(t)\right)+u_{k}(t, r(t))\right] \mathrm{d} t \\
& +g_{k}\left(e_{k}(t), t, r(t)\right) \mathrm{d} W(t), \quad k=1,2, \ldots, N .
\end{aligned}
$$

Apparently, for any $i \in \mathbb{S}, F_{k}(0, t, i)=0, k=1,2, \ldots, N$ and $H_{s}(0, t, i)=0, s=1,2, \ldots, l$. We assume that $g_{k}(0, t, i)=0, k=1,2, \ldots, N$, for any $i \in \mathbb{S}$ and $t \geq t_{0}$, which means that $e(t)=0$ is the trivial solution of error system (3). For any given initial values $e\left(t_{0}\right)=e_{0} \in \mathbb{R}^{n N}$ and $r\left(t_{0}\right)=r_{0} \in \mathbb{S}$, the solution of (3) can be expressed by $e\left(t ; t_{0}, e_{0}, r_{0}\right)$. In most cases, write $e\left(t ; t_{0}, e_{0}, r_{0}\right)$ as $e(t)$ for simplicity. If $r(t)=i \in \mathbb{S}$, then for $V_{k}\left(e_{k}, t, i\right) \in C^{2,1}\left(\mathbb{R}^{n} \times \mathbb{R}_{+}^{1} \times \mathbb{S}\right.$; $\left.\mathbb{R}_{+}^{1}\right)$, define the differential operator $\mathcal{L} V_{k}\left(e_{k}, t, i\right)$ associated with the $k$ th equation of error 
system (3) by

$$
\begin{aligned}
\mathcal{L} V_{k}\left(e_{k}, t, i\right) \triangleq & \sum_{j=1}^{m} \gamma_{i j} V_{k}\left(e_{k}, t, j\right)+\frac{\partial V_{k}\left(e_{k}, t, i\right)}{\partial t}+\frac{\partial V_{k}\left(e_{k}, t, i\right)}{\partial e_{k}}\left[F_{k}\left(e_{k}(t), t, i\right)\right. \\
& +\delta_{1}(i) \sum_{h=1}^{N} b_{k h}^{1}(i) H_{1}\left(e_{h}(t), t, i\right)+\delta_{2}(i) \sum_{h=1}^{N} b_{k h}^{2}(i) H_{2}\left(e_{h}(t), t, i\right)+\cdots \\
& \left.+\delta_{l}(i) \sum_{h=1}^{N} b_{k h}^{l}(i) H_{l}\left(e_{h}(t), t, i\right)+u_{k}(t, i)\right] \\
& +\frac{1}{2} \operatorname{trace}\left\{g_{k}^{\mathrm{T}}\left(e_{k}, t, i\right) \frac{\partial^{2} V_{k}\left(e_{k}, t, i\right)}{\partial e_{k}^{2}} g_{k}\left(e_{k}, t, i\right)\right\},
\end{aligned}
$$

where

$$
\begin{aligned}
& \frac{\partial V_{k}\left(e_{k}, t, i\right)}{\partial e_{k}}=\left(\frac{\partial V_{k}\left(e_{k}, t, i\right)}{\partial e_{k}^{(1)}}, \frac{\partial V_{k}\left(e_{k}, t, i\right)}{\partial e_{k}^{(2)}}, \ldots, \frac{\partial V_{k}\left(e_{k}, t, i\right)}{\partial e_{k}^{(n)}}\right), \\
& \frac{\partial^{2} V_{k}\left(e_{k}, t, i\right)}{\partial e_{k}^{2}}=\left(\frac{\partial^{2} V_{k}\left(e_{k}, t, i\right)}{\partial e_{k}^{(d)} \partial e_{k}^{(b)}}\right)_{n \times n} .
\end{aligned}
$$

We recall the definitions about exponential synchronization and asymptotical synchronization of drive-response networks (1) and (2) given in [44].

Definition 1 The drive-response networks (1) and (2) are said to be $p$ th moment exponentially synchronized if for any $\varepsilon>0$, there exist positive constants $a$ and $\delta$ such that

$$
\mathbb{E}\left|e_{0}\right|^{p} \leq \delta \quad \Longrightarrow \quad \mathbb{E}\left|e\left(t ; t_{0}, e_{0}, r_{0}\right)\right|^{p} \leq \varepsilon e^{-a\left(t-t_{0}\right)}, \quad t \geq t_{0}
$$

for some $p>0$ and all $\left(t_{0}, e_{0}, r_{0}\right) \in \mathbb{R}_{+}^{1} \times \mathbb{R}^{n N} \times \mathbb{S}$. When $p=2$, they are said to be exponentially synchronized in mean square. Moreover, the drive-response networks (1) and (2) are said to be almost surely exponentially synchronized if

$$
\limsup _{t \rightarrow \infty} \frac{1}{t} \log \left|e\left(t ; t_{0}, e_{0}, r_{0}\right)\right|<0 \quad \text { a.s. }
$$

for all $\left(t_{0}, e_{0}, r_{0}\right) \in \mathbb{R}_{+}^{1} \times \mathbb{R}^{n N} \times \mathbb{S}$.

Definition 2 The drive-response networks (1) and (2) are said to be asymptotically synchronized in $p$ th moment if

$$
\lim _{t \rightarrow \infty} \mathbb{E}\left(\left|e\left(t ; t_{0}, e_{0}, r_{0}\right)\right|^{p}\right)=0
$$

for all $\left(t_{0}, e_{0}, r_{0}\right) \in \mathbb{R}_{+}^{1} \times \mathbb{R}^{n N} \times \mathbb{S}$ and some $p>0$. When $p=2$, they are said to be asymptotically synchronized in mean square. Moreover, they are said to be almost surely asymptotically synchronized or asymptotically synchronized with probability 1 if

$$
\lim _{t \rightarrow \infty} e\left(t ; t_{0}, e_{0}, r_{0}\right)=0 \quad \text { a.s. }
$$

for all $\left(t_{0}, e_{0}, r_{0}\right) \in \mathbb{R}_{+}^{1} \times \mathbb{R}^{n N} \times \mathbb{S}$. 
Finally, we state two important lemmas from graph theory. We refer the readers to [37, 45] for some basic concepts of graph theory.

Lemma 1 (Kirchhoff's matrix-tree theorem) Let $N \geq 2$, and let $(\mathcal{G}, A(i)), A(i)=$ $\left(a_{k h}(i)\right)_{N \times N}$, be a weighted digraph for all $i \in \mathbb{S}$, and let $c_{k}(i)$ be the cofactor of the kth diagonal element of the Laplacian matrix of $(\mathcal{G}, A(i))$. Then

$$
c_{k}(i)=\sum_{\mathcal{T} \in \mathbb{T}_{k}(i)} \omega(\mathcal{T}), \quad k=1,2, \ldots, N, i \in \mathbb{S},
$$

where $\mathbb{T}_{k}(i)$ is the set of all spanning trees $\mathcal{T}$ of $(\mathcal{G}, A(i))$ that are rooted at vertex $k$, and $\omega(\mathcal{T})$ is the weight of $\mathcal{T}$. Additionally, if $(\mathcal{G}, A(i))$ is strongly connected, then $c_{k}(i)>0$ for $k=1,2, \ldots, N, i \in \mathbb{S}$.

Lemma 2 Let $N \geq 2$, and let $(\mathcal{G}, A(i)), A(i)=\left(a_{k h}(i)\right)_{N \times N}$, be a weighted digraph for all $i \in \mathbb{S}$. Let $\mathbb{Q}$ be the set of all spanning unicyclic graphs $\mathcal{Q}$ of $(\mathcal{G}, A(i)), C_{\mathcal{Q}}$ be the cycle of $\mathcal{Q}$, $\omega(\mathcal{Q})$ be the weight of $\mathcal{Q}$, and let $c_{k}(i)$ be defined in Lemma 1 . Then for arbitrary functions $F_{k h}\left(e_{k}, e_{h}, t, i\right), k, h=1,2, \ldots, N$,

$$
\sum_{k, h=1}^{N} c_{k}(i) a_{k h}(i) F_{k h}\left(e_{k}, e_{h}, t, i\right)=\sum_{\mathcal{Q} \in \mathbb{Q}} \omega(\mathcal{Q}) \sum_{(q, r) \in E\left(C_{\mathcal{Q}}\right)} F_{r q}\left(e_{r}, e_{q}, t, i\right), \quad i \in \mathbb{S} .
$$

Lemmas 1 and 2 can be easily obtained by the methods similar to those in Proposition 2.1 and Theorem 2.2 in [37], respectively. So we omit their proofs.

\section{Synchronization analysis for drive-response networks (1) and (2)}

In this section, we are proceeding to investigate exponential synchronization and asymptotical synchronization of drive-response networks (1) and (2). Motivated by the research method in $[22,26]$, we will obtain some sufficient criteria by using some results in graph theory and the Lyapunov method. Here we construct the following state feedback controller for the response system (2):

$$
u_{k}(t, r(t))=\bar{b}_{k}\left[f_{k}\left(y_{k}(t), t, r(t)\right)-f_{k}\left(x_{k}(t), t, r(t)\right)\right] \text {, }
$$

in which $\bar{b}_{k} \neq-1$ is the gain constant to be designed. The error network (3) is turned into

$$
\begin{aligned}
\mathrm{d} e_{k}(t)= & {\left[\left(1+\bar{b}_{k}\right) F_{k}\left(e_{k}(t), t, r(t)\right)+\delta_{1}(r(t)) \sum_{h=1}^{N} b_{k h}^{1}(r(t)) H_{1}\left(e_{h}(t), t, r(t)\right)\right.} \\
& +\delta_{2}(r(t)) \sum_{h=1}^{N} b_{k h}^{2}(r(t)) H_{2}\left(e_{h}(t), t, r(t)\right)+\cdots \\
& \left.+\delta_{l}(r(t)) \sum_{h=1}^{N} b_{k h}^{l}(r(t)) H_{l}\left(e_{h}(t), t, r(t)\right)\right] \mathrm{d} t \\
& +g_{k}\left(e_{k}(t), t, r(t)\right) \mathrm{d} W(t), \quad k=1,2, \ldots, N .
\end{aligned}
$$

We begin by stating some basic assumptions. 
$A 1$. For any $k \in\{1,2, \ldots, N\}$ and $i \in \mathbb{S}$, there exist positive constants $\alpha_{k}(i), \beta_{k}(i)$, and $\gamma_{k}(i)$, functions $V_{k}\left(e_{k}, t, i\right)$ and $F_{k h}\left(e_{k}, e_{h}, t, i\right)$, and a matrix $A(i)=\left(a_{k h}(i)\right)_{N \times N}, a_{k h}(i) \geq 0$, such that

$$
\alpha_{k}(i)\left|e_{k}\right|^{p} \leq V_{k}\left(e_{k}, t, i\right) \leq \beta_{k}(i)\left|e_{k}\right|^{p}
$$

and

$$
\begin{aligned}
\mathcal{L} V_{k}\left(e_{k}, t, i\right) \leq & -\gamma_{k}(i)\left|e_{k}\right|^{p} \\
& +\sum_{h=1}^{N} a_{k h}(i) F_{k h}\left(e_{k}, e_{h}, t, i\right), \quad e_{k}, e_{h} \in \mathbb{R}^{n}, t \geq t_{0}, i \in \mathbb{S} .
\end{aligned}
$$

$A 2$. Along each directed cycle $\mathcal{C}$ of the weighted digraph $(\mathcal{G}, A(i))$, we have

$$
\sum_{(h, k) \in E(\mathcal{C})} F_{k h}\left(e_{k}, e_{h}, t, i\right) \leq 0, \quad e_{k}, e_{h} \in \mathbb{R}^{n}, t \geq t_{0}, i \in \mathbb{S}
$$

Remark 1 Assumption $A 2$ holds for each directed cycle $C$ and any $e_{k}, e_{h}$, which seems difficult to be checked. Actually, we do not need to verify it directly. We just need to find some appropriate functions. For example, assume that there exist functions $q_{k}(t, i), k=$ $1,2, \ldots, N, i \in \mathbb{S}$, such that

$$
F_{k h}\left(e_{k}, e_{h}, t, i\right) \leq q_{k}(t, i)-q_{h}(t, i), \quad k, h=1,2, \ldots, N, i \in \mathbb{S} .
$$

Then we can obtain that

$$
\sum_{(h, k) \in E(C)} F_{k h}\left(e_{k}, e_{h}, t, i\right) \leq \sum_{(h, k) \in E(C)}\left(q_{k}(t, i)-q_{h}(t, i)\right)=0
$$

Theorem 1 Let $(\mathcal{G}, A(i))$ be strongly connected for any $i \in \mathbb{S}$. If assumptions $A 1$ and $A 2$ hold, then there exist constants $C$ and positive constants $\eta$ such that

$$
\mathbb{E}\left|e\left(t ; t_{0}, e_{0}, r_{0}\right)\right|^{p} \leq C\left|e_{0}\right|^{p} e^{-\eta\left(t-t_{0}\right)}, \quad t \geq t_{0},
$$

for some $p>0$. That is, the drive-response networks (1) and (2) are pth moment exponentially synchronized under controller (5).

Proof Let $V(e, t, i)=\sum_{k=1}^{N} c_{k}(i) V_{k}\left(e_{k}, t, i\right)$, where $c_{k}(i)$ is defined as in Lemma 1 . Then by applying the research method in [26] there exist positive constants $\alpha$ and $\beta$ such that

$$
\alpha|e|^{p} \leq V(e, t, i) \leq \beta|e|^{p}, \quad i \in \mathbb{S} .
$$


Fix any $\left(t_{0}, e_{0}, r_{0}\right) \in \mathbb{R}_{+}^{1} \times \mathbb{R}^{n N} \times \mathbb{S}$ and write $e\left(t ; t_{0}, e_{0}, r_{0}\right)$ as $e(t)$ for simplicity. For fixed $t>t_{0}$, since $\omega(\mathcal{Q})>0$, from assumption $A 2$ and Lemma 2 , we can deduce that

$$
\begin{aligned}
\mathcal{L} V(e, t, i) & =\sum_{k=1}^{N} c_{k}(i) \mathcal{L} V_{k}\left(e_{k}, t, i\right) \\
& \leq \sum_{k=1}^{N} c_{k}(i)\left(-\gamma_{k}(i)\left|e_{k}\right|^{p}+\sum_{h=1}^{N} a_{k h}(i) F_{k h}\left(e_{k}, e_{h}, t, i\right)\right) \\
& =-\sum_{k=1}^{N} c_{k}(i) \gamma_{k}(i)\left|e_{k}\right|^{p}+\sum_{\mathcal{Q} \in \mathbb{Q}} \omega(\mathcal{Q}) \sum_{(h, k) \in E\left(C_{\mathcal{Q}}\right)} F_{k h}\left(e_{k}, e_{h}, t, i\right) \\
& \leq-\min _{1 \leq k \leq N}\left\{c_{k}(i) \gamma_{k}(i)\right\} \sum_{k=1}^{N}\left|e_{k}\right|^{p} \\
& \leq-\min _{1 \leq k \leq N}\left\{c_{k}(i) \gamma_{k}(i)\right\} N^{\left(1-\frac{p}{2}\right) \wedge 0}|e|^{p} \triangleq-\lambda|e|^{p} .
\end{aligned}
$$

Since $e_{0}$ is given, a large positive number $d_{0}$ can be chosen to satisfy $\left|e_{0}\right|^{p} \leq d_{0}$. For any integer $d>d_{0}$, define the stopping time

$$
\sigma_{d}=\inf \left\{t \geq t_{0}:|e(t)| \geq d\right\}
$$

and set $\inf \emptyset=\infty$ as usual. Moreover, by Itô formula (see [44]) we have

$$
\begin{aligned}
\mathbb{E} & {\left[e^{\frac{\lambda}{\beta}\left(t \wedge \sigma_{d}\right)} V\left(e\left(t \wedge \sigma_{d}\right), t \wedge \sigma_{d}, r\left(t \wedge \sigma_{d}\right)\right)\right] } \\
& =\mathbb{E} V\left(e_{0}, t_{0}, r_{0}\right) e^{\frac{\lambda}{\beta} t_{0}}+\mathbb{E} \int_{t_{0}}^{t \wedge \sigma_{d}} e^{\frac{\lambda}{\beta} s}\left[\mathcal{L} V(e(s), s, r(s))+\frac{\lambda}{\beta} V(e(s), s, r(s))\right] \mathrm{d} s \\
& \leq \beta\left|e_{0}\right|^{p} e^{\frac{\lambda}{\beta} t_{0}}+\mathbb{E} \int_{t_{0}}^{t \wedge \sigma_{d}} e^{\frac{\lambda}{\beta} s}\left[-\lambda|e(s)|^{p}+\frac{\lambda}{\beta} \beta|e(s)|^{p}\right] \mathrm{d} s \\
& =\beta\left|e_{0}\right|^{p} e^{\frac{\lambda}{\beta} t_{0}} .
\end{aligned}
$$

By applying the research method in [43] it is straightforward to show that

$$
\mathbb{E}|e(t)|^{p} \leq \alpha^{-1} \beta\left|e_{0}\right|^{p} e^{-\frac{\lambda}{\beta}\left(t-t_{0}\right)}
$$

Therefore, for any $\varepsilon>0$, there exists a positive constant $\delta=\varepsilon /\left(\alpha^{-1} \beta\right)$ such that

$$
\mathbb{E}\left|e_{0}\right|^{p} \leq \delta \quad \Longrightarrow \quad \mathbb{E}\left|e\left(t, e_{0}, r_{0}\right)\right|^{p} \leq \varepsilon e^{-\frac{\lambda}{\beta}\left(t-t_{0}\right)}, t \geq t_{0}
$$

In other words, two coupled networks (1) and (2) can be $p$ th moment exponentially synchronized. The proof is completed.

Remark 2 If we adopt Lyapunov stability theory, then it is vital to construct an appropriate Lyapunov function for the system. However, in our model, large number of nodes in the networks, multi-weights among nodes, and stochastic perturbations are all considered, which can make the construction of a Lyapunov function very difficult. Just for 
this, very few researchers have studied the dynamical behavior of the multi-weighted coupled networks with Markovian switching. Especially, the problem of synchronization for multi-weighted complex dynamical networks with Markovian switching has not yet been studied. With the help of some results in graph theory such as Kirchhoff's matrixtree theorem, a global Lyapunov function of the error network (6) can be easily constructed, which is a weighted sum of the Lyapunov functions of vertex systems, that is, $V(e, t, i)=\sum_{k=1}^{N} c_{k}(i) V_{k}\left(e_{k}, t, i\right)$.

Remark 3 The related results in the literature are divided into two parts: one is mainly about the synchronization of complex networks with multiple weights but without stochastic perturbations [31-34,36], whereas the other part is about the synchronization of stochastic complex networks with single weight $[12,26]$. Only few papers investigated the synchronization of stochastic multiple complex networks with white noise or Lévy noise [22, 29, 35, 46]. We focus on the synchronization of stochastic multiple complex networks with white noise and telegraph noise. The model is different from the results in the literature. The most important in this paper is that a novel graph-theoretic approach and stochastic analysis are combined to get the synchronization criteria, which is also different from the results in the literature.

In Theorem 1, some synchronized conditions are given in the form of Lyapunov functions of vertex systems. In fact, we need to find some appropriate Lyapunov functions to check the conditions of Theorem 1, which is the task of Theorem 2. The following assumptions on the coefficients of the error system (6) are imposed for any $k=1,2, \ldots, N$, $e_{k} \in \mathbb{R}^{n}, t \geq t_{0}$, and $i \in \mathbb{S}$.

A3. There are constants $\sigma_{k}(i)$ and $\rho_{k}(i) \geq 0$ such that

$$
e_{k}^{\mathrm{T}} F_{k}\left(e_{k}, t, i\right) \leq \sigma_{k}(i)\left|e_{k}\right|^{2}, \quad\left|g_{k}\left(e_{k}, t, i\right)\right|^{2} \leq \rho_{k}(i)\left|e_{k}\right|^{2} .
$$

A4. There exists a positive number $\eta_{s}(i)$ such that

$$
\left|H_{s}\left(e_{k}, t, i\right)\right| \leq \eta_{s}(i)\left|e_{k}\right|, \quad s=1,2, \ldots, l .
$$

For brevity, we set

$$
D_{k h}(i)=\max _{1 \leq s \leq l}\left\{\delta_{s}(i) b_{k h}^{s}(i) \eta_{s}(i)\right\}, \quad k, h=1,2, \ldots, N, i \in \mathbb{S}
$$

For each $p \geq 2$ and $k=1,2, \ldots, N$, define the $m \times m$ matrix

$$
\mathcal{A}_{k}(p):=-\operatorname{diag}\left(M_{k}(1)+p l \sum_{h=1}^{N} D_{k h}(1), \ldots, M_{k}(m)+p l \sum_{h=1}^{N} D_{k h}(m)\right)-\Gamma
$$

where

$$
M_{k}(i)=p\left|1+\bar{b}_{k}\right| \sigma_{k}(i)+\frac{1}{2} p(p-1) \rho_{k}(i) .
$$


Theorem 2 Suppose that assumptions $A 3, A 4$ hold and $\left(\mathcal{G},\left(D_{k h}(i)\right)_{N \times N}\right)$ is strongly connected for each $i \in \mathbb{S}$. If $\mathcal{A}_{k}(p)$ is a nonsingular $M$-matrix for any $k=1,2, \ldots, N$ and some $p \geq 2$, then the drive-response networks (1) and (2) are pth moment exponentially synchronized under controller (5).

Proof For simplicity, we write $F_{k}\left(e_{k}(t), t, r(t)\right)=F_{k}, H_{s}\left(e_{h}, t, i\right)=H_{s}$ and $g_{k}\left(e_{k}, t, i\right)=g_{k}$. Since $\mathcal{A}_{k}(p)$ is a nonsingular M-matrix, we can find $q_{k}(i)>0$ and $\lambda_{k}(i)>0$ such that $\lambda_{k}=\mathcal{A}_{k}(p) q_{k}$, where $\lambda_{k}=\left(\lambda_{k}(1), \lambda_{k}(2), \ldots, \lambda_{k}(m)\right)^{\mathrm{T}}$ and $q_{k}=\left(q_{k}(1), q_{k}(2), \ldots, q_{k}(m)\right)^{\mathrm{T}}$. Define the function $V_{k}: \mathbb{R}^{n} \times \mathbb{S} \rightarrow \mathbb{R}_{+}^{1}$ by $V_{k}\left(e_{k}, i\right)=q_{k}(i)\left|e_{k}\right|^{p}$. Now we can compute $\mathcal{L} V_{k}\left(e_{k}, i\right)$ by the definition of operator (4):

$$
\begin{aligned}
& \mathcal{L} V_{k}\left(e_{k}, i\right) \\
& \left.=q_{k}(i) p\left|e_{k}\right|^{p-2} e_{k}^{\mathrm{T}}(t)\left[\left(1+\bar{b}_{k}\right) F_{k}+\delta_{1}(i) \sum_{h=1}^{N} b_{k h}^{1}(i) H_{1}+\cdots+\delta_{l}(i) \sum_{h=1}^{N} b_{k h}^{l}(i)\right) H_{l}\right] \\
& +\sum_{j=1}^{m} \gamma_{i j} q_{k}(j)\left|e_{k}\right|^{p}+\frac{1}{2} \operatorname{trace}\left[g_{k}^{\mathrm{T}}\left(q_{k}(i) p\left|e_{k}\right|^{p-2} I+q_{k}(i) p(p-2)\left|e_{k}\right|^{p-4} e_{k} e_{k}^{\mathrm{T}}\right) g_{k}\right] \\
& \leq q_{k}(i) p\left|1+\bar{b}_{k}\right| \sigma_{k}(i)\left|e_{k}\right|^{p} \\
& +q_{k}(i) p\left|e_{k}\right|^{p-1}\left[\delta_{1}(i) \sum_{h=1}^{N} b_{k h}^{1}(i) \eta_{1}(i)\left|e_{h}\right|+\cdots+\delta_{l}(i) \sum_{h=1}^{N} b_{k h}^{l}(i) \eta_{l}(i)\left|e_{h}\right|\right] \\
& +\sum_{j=1}^{m} \gamma_{i j} q_{k}(j)\left|e_{k}\right|^{p}+\frac{1}{2} q_{k}(i) p\left|e_{k}\right|^{p-2}\left|g_{k}\right|^{2}+\frac{1}{2} q_{k}(i) p(p-2)\left|e_{k}\right|^{p-4}\left|e_{k}^{\mathrm{T}} g_{k}\right|^{2} \\
& \leq\left[q_{k}(i) p\left|1+\bar{b}_{k}\right| \sigma_{k}(i)+\sum_{j=1}^{m} \gamma_{i j} q_{k}(j)+\frac{1}{2} q_{k}(i) p(p-1) \rho_{k}(i)\right]\left|e_{k}\right|^{p} \\
& +q_{k}(i) p \sum_{h=1}^{N} \delta_{1}(i) b_{k h}^{1}(i) \eta_{1}(i)\left(\frac{p-1}{p}\left|e_{k}\right|^{p}+\frac{1}{p}\left|e_{h}\right|^{p}\right) \\
& +\cdots+q_{k}(i) p \sum_{h=1}^{N} \delta_{l}(i) b_{k h}^{l}(i) \eta_{l}(i)\left(\frac{p-1}{p}\left|e_{k}\right|^{p}+\frac{1}{p}\left|e_{h}\right|^{p}\right) \\
& \leq\left[q_{k}(i) p\left|1+\bar{b}_{k}\right| \sigma_{k}(i)+\sum_{j=1}^{m} \gamma_{i j} q_{k}(j)+\frac{1}{2} q_{k}(i) p(p-1) \rho_{k}(i)\right]\left|e_{k}\right|^{p} \\
& +q_{k}(i) p l \sum_{h=1}^{N} D_{k h}(i)\left(\frac{p-1}{p}\left|e_{k}\right|^{p}+\frac{1}{p}\left|e_{h}\right|^{p}\right) \\
& \leq\left[q_{k}(i) p\left|1+\bar{b}_{k}\right| \sigma_{k}(i)+\sum_{j=1}^{m} \gamma_{i j} q_{k}(j)+\frac{1}{2} q_{k}(i) p(p-1) \rho_{k}(i)+q_{k}(i) p l \sum_{h=1}^{N} D_{k h}(i)\right]\left|e_{k}\right|^{p} \\
& +q_{k}(i) l \sum_{h=1}^{N} D_{k h}(i)\left(\left|e_{h}\right|^{p}-\left|e_{k}\right|^{p}\right) \\
& =-\lambda_{k}(i)\left|e_{k}\right|^{p}+\sum_{h=1}^{N} q_{k}(i) D_{k h}(i) l\left(\left|e_{h}\right|^{p}-\left|e_{k}\right|^{p}\right) \triangleq-\lambda_{k}(i)\left|e_{k}\right|^{p}+\sum_{h=1}^{N} a_{k h}(i) F_{k h}\left(e_{k}, e_{h}, t, i\right),
\end{aligned}
$$


where $a_{k h}(i)=q_{k}(i) D_{k h}(i)$ and $F_{k h}\left(e_{k}, e_{h}, t, i\right)=l\left(\left|e_{h}\right|^{p}-\left|e_{k}\right|^{p}\right)$. Clearly, for each $i \in \mathbb{S}$, the strong connectedness of $\left(\mathcal{G},\left(D_{k h}(i)\right)_{N \times N}\right)$ implies the strong connectedness of $\left(\mathcal{G},\left(a_{k h}(i)\right)_{N \times N}\right)$. Furthermore, along each directed cycle $\mathcal{C}$ of weighted digraph $(\mathcal{G}, A(i))$ $\left(A(i)=\left(a_{k h}(i)\right)_{N \times N}\right)$, it follows that

$$
\sum_{(h, k) \in E(\mathcal{C})} F_{k h}\left(e_{k}, e_{h}, t, i\right)=\sum_{(h, k) \in E(\mathcal{C})} l\left(\left|e_{h}\right|^{p}-\left|e_{k}\right|^{p}\right)=0 .
$$

All conditions in Theorem 1 have been checked. Thus drive-response networks (1) and (2) are $p$ th moment exponentially synchronized, which completes the proof.

Remark 4 In Theorem 2, $p$ th moment exponential synchronization of drive-response networks (1) and (2) has been obtained by combining a graph-theoretic method and M-matrix theory. The obtained synchronization criteria are closely related to the coupling strength $\delta_{s}$ of each subnetwork, inner coupling $\bar{H}_{s}$, multi-weights $b_{k h}^{s}$, the perturbation intensity $g_{k}$ of white noise, and the generator $\Gamma$ of Markov chain. Theorem 2 is shown with the coefficients of drive-response networks (1) and (2), whereas Theorem 1 is given by Lyapunov functions. Theorem 1 is the theoretical basis of Theorem 2, but Theorem 2 can be much more easily checked than Theorem 1.

In Theorem 2, $\left(\mathcal{G},\left(D_{k h}(i)\right)_{N \times N}\right)$ is strongly connected for each $i \in \mathbb{S}$. Define $B_{k h}(i)=$ $\max _{1 \leq s \leq l}\left\{b_{k h}^{s}(i)\right\}$. Since $\delta_{s}(i)>0$ and $\eta_{s}(i)>0$, the strong connectedness of $\left(\mathcal{G},\left(B_{k h}(i)\right)_{N \times N}\right)$ implies the strong connectedness of $\left(\mathcal{G},\left(D_{k h}(i)\right)_{N \times N}\right)$. In other words, we do not need the strong connectedness of each subnetwork $\left(\mathcal{G},\left(b_{k h}(i)\right)_{N \times N}\right)$ to ensure the synchronization of drive-response networks (1) and (2).

Remark 5 In [26] the exponential synchronization criteria of single-weighted stochastic coupled networks with Markovian switching have been obtained. However, in this paper, we focus on the multi-weighted stochastic coupled networks with Markovian switching. The inclusion of multi-weights makes coupled networks being split into multiple subnetworks. Hence we cannot directly use the theory in [26] to solve the problem. In $[22,43]$ the synchronization and stability of stochastic multi-weighted coupled networks with white noise have been obtained by the graph-theoretic method. In our paper, the perturbations by white noise and color noise are both considered in the multi-weighted coupled networks. Our model is more general, and the method is novel. Compared with the existing methods in the literature, we successfully construct a Lyapunov function for SMWCNMS indirectly. In more detail, by the graph-theoretic method we can easily construct a Lyapunov function for SMWCNMS as the weighted sum of Lyapunov functions of vertex systems, that is, $V(e, t, i)=\sum_{k=1}^{N} c_{k}(i) V_{k}\left(e_{k}, t, i\right)$, where $c_{k}(i)$ is the cofactor of the $k$ th diagonal element of the Laplacian matrix of $(\mathcal{G}, A(i)),\left(A(i)=\left(a_{k h}(i)\right)_{N \times N}\right)=\left(q_{k}(i) D_{k h}(i)\right)_{N \times N}$.

Corollary 1 The drive-response networks (1) and (2) are almost surely exponentially synchronized under the conditions of Theorem 2.

This corollary can be proved by applying the Borel-Cantelli lemma and BurkholderDavis-Gundy inequality. We omit its proof for simplicity. For more detail, see [44].

Until now, we have shown the exponential synchronization of drive-response networks (1) and (2). In the real applications, there exists another kind of synchronization, that is, asymptotical synchronization. 
Theorem 3 Under the conditions of Theorem 2, the drive-response networks (1) and (2) are not only asymptotically synchronized in pth moment, but also almost surely asymptotically synchronized.

Motivated by [44], before proving Theorem 3, we show a lemma.

Lemma 3 Assume that there are functions $V \in C^{2,1}\left(\mathbb{R}^{n N} \times \mathbb{R}_{+}^{1} \times \mathbb{S} ; \mathbb{R}_{+}^{1}\right)$ and $\gamma \in L^{1}\left(\mathbb{R}_{+}^{1} ; \mathbb{R}_{+}^{1}\right)$ and positive constants $p$ and $v$ such that

$$
\mathcal{L} V(e, t, i) \leq \gamma(t)-v|e|^{p}
$$

Moreover, assume that there exist positive constants $L$ and $M$ such that

$$
\left|F_{k}\left(e_{k}, t, i\right)\right|+\left|g_{k}\left(e_{k}, t, i\right)\right| \leq L\left|e_{k}\right|, \quad\left|H_{s}\left(e_{k}, t, i\right)\right| \leq M\left|e_{k}\right|, \quad k=1,2, \ldots, l,
$$

for all $\left(e_{k}, t, i\right) \in \mathbb{R}^{n} \times \mathbb{R}_{+}^{1} \times \mathbb{S}$. Then the drive-response networks (1) and (2) are not only asymptotically synchronized in pth moment, but also almost surely asymptotically synchronized.

Proof Obviously, under condition (15), there exist positive constants $C$ and $D$ such that for all $k=1,2, \ldots, N$ and $i \in \mathbb{S}$,

$$
\left|g_{k}\left(e_{k}(t), t, i\right)\right| \leq D\left|e_{k}(t)\right|
$$

and

$$
\begin{aligned}
& \left|\left(1+\bar{b}_{k}\right) F_{k}\left(e_{k}(t), t, i\right)+\delta_{1}(i) \sum_{h=1}^{N} b_{k h}^{1}(i) H_{1}\left(e_{h}(t), t, i\right)+\cdots+\delta_{l}(i) \sum_{h=1}^{N} b_{k h}^{l}(i) H_{l}\left(e_{h}(t), t, i\right)\right| \\
& \quad \leq C\left|e_{k}(t)\right| .
\end{aligned}
$$

Then the conditions of Theorem 5.29 in [44] can be easily checked for the error network (6). So, the error network (6) is not only asymptotically stable in the $p$ th moment, but also almost surely asymptotically stable. In other words, the drive-response networks (1) and (2) are not only asymptotically synchronized in $p$ th moment, but also almost surely asymptotically synchronized.

Now let us prove Theorem 3 .

Proof Define

$$
V_{k}\left(e_{k}, i\right)=q_{k}(i)\left|e_{k}\right|^{p}
$$

where $q_{k}(i)$ is defined in Theorem 2 . From the proof of Theorem 2 we can obtain that

$$
\mathcal{L} V_{k}\left(e_{k}, i\right) \leq-\lambda_{k}(i)\left|e_{k}\right|^{p}+\sum_{h=1}^{N} q_{k}(i) D_{k h}(i) l\left(\left|e_{h}\right|^{p}-\left|e_{k}\right|^{p}\right)
$$


Define $V(e, i)=\sum_{k=1}^{N} c_{k}(i) V_{k}\left(e_{k}, i\right)$, where $c_{k}(i)$ is the cofactor of the $k$ th diagonal element of the Laplacian matrix of $(\mathcal{G}, A(i)), A(i)=\left(q_{k}(i) D_{k h}(i)\right)_{N \times N}$. Then we can obtain from Theorem 1 that there exists a positive constant $\bar{\lambda}$ such that

$$
\mathcal{L} V(e, i) \leq-\bar{\lambda}|e|^{p}
$$

From assumptions $A 3$ and $A 4$, it is easy to find positive numbers $L$ and $M$ to meet (15). Until now, all conditions of Lemma 3 have been checked. Hence Lemma 3 tells us that drive-response networks (1) and (2) can be asymptotically synchronized in $p$ th moment and almost surely asymptotically synchronized.

\section{Numerical example}

To illustrate the theoretical results, we give a numerical example.

Example 1 Let $r(t)$ be a right-continuous Markovian chain taking values in $\mathbb{S}=\{1,2\}$ with generator

$$
\Gamma=\left(\gamma_{i j}\right)_{2 \times 2}=\left(\begin{array}{cc}
-1 & 1 \\
7 & -7
\end{array}\right) .
$$

For brevity, consider a network $\mathcal{G}$ with six nodes and three different kinds of weights in Fig. 1. In the web version of this paper, the readers can see three kinds of weights more clearly. The following coupled system with Markovian switching on $\mathcal{G}$ is designed as a drive system:

$$
\begin{aligned}
\dot{x}_{k}(t)= & \frac{\alpha(r(t))}{k} x_{k}(t)+\delta_{1}(r(t)) \sum_{h=1}^{6} b_{k h}^{1}(r(t)) \mathbf{A}(r(t)) \cos \left(x_{h}(t)\right) \\
& +\delta_{2}(r(t)) \sum_{h=1}^{6} b_{k h}^{2}(r(t)) \mathbf{B}(r(t)) \sin \left(x_{h}(t)\right) \\
& +\delta_{3}(r(t)) \sum_{h=1}^{6} b_{k h}^{3}(r(t)) \mathbf{C}(r(t)) x_{h}(t), \quad k=1,2, \ldots, 6,
\end{aligned}
$$

where $x_{k} \in \mathbb{R}^{3}, r(t) \in \mathbb{S}=\{1,2\}, \alpha(1)=-0.6, \alpha(2)=-1.2, \delta_{1}(1)=0.4, \delta_{2}(1)=0.25, \delta_{3}(1)=$ $0.2, \delta_{1}(2)=0.5, \delta_{2}(2)=1, \delta_{3}(2)=0.5$, and $\mathbf{A}(1)=\operatorname{diag}(0.5,0.4,0.3), \mathbf{B}(1)=\operatorname{diag}(0.8,0.6,0.5)$, $\mathbf{C}(1)=\operatorname{diag}(1,0.5,0.6), \quad \mathbf{A}(2)=\operatorname{diag}(0.8,0.6,0.5), \quad \mathbf{B}(2)=\operatorname{diag}(0.3,0.2,0.4), \mathbf{C}(2)=$ $\operatorname{diag}(0.4,0.8,0.5)$.

The corresponding noise-perturbed response system is described by

$$
\begin{aligned}
\mathrm{d} y_{k}(t)= & {\left[\frac{\alpha(r(t))}{k} y_{k}(t)+\delta_{1}(r(t)) \sum_{h=1}^{6} b_{k h}^{1}(r(t)) \mathbf{A}(r(t)) \cos \left(y_{h}(t)\right)\right.} \\
& +\delta_{2}(r(t)) \sum_{h=1}^{6} b_{k h}^{2}(r(t)) \mathbf{B}(r(t)) \sin \left(y_{h}(t)\right)+\delta_{3}(r(t)) \sum_{h=1}^{6} b_{k h}^{3}(r(t)) \mathbf{C}(r(t)) y_{h}(t) \\
& \left.+u_{k}(t, r(t))\right] \mathrm{d} t+\beta_{k}(r(t))\left(y_{k}(t)-x_{k}(t)\right) \mathrm{d} W(t), \quad k=1,2, \ldots, 6
\end{aligned}
$$




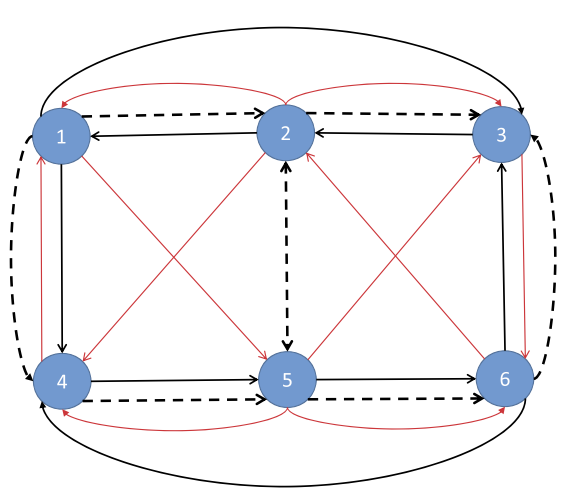

(a) State 1



(b) State 2

Figure 1 The diagram for a network with six nodes, three different kinds of weights, and two states

where $W(t)$ is a scalar Brownian motion, $\beta_{k}(1)=(0.02 k)^{1 / 2}, \beta_{k}(2)=(0.01 k)^{1 / 2}, k=$ $1,2, \ldots, 6$. The state feedback controller is designed as

$$
u_{k}(t, r(t))=\bar{b}_{k}\left[\frac{\alpha(r(t))}{k} y_{k}(t)-\frac{\alpha(r(t))}{k} x_{k}(t)\right], \quad k=1,2, \ldots, 6,
$$

where $\bar{b}_{k} \neq-1$ is the gain constant to be scheduled. Three weighted matrices in two states $Q^{s}(i)=\left(b_{k h}^{s}(i)\right)_{6 \times 6}(s=1,2,3, i=1,2)$ are given as follows:

$$
\begin{aligned}
Q^{1}(1) & =\left(\begin{array}{cccccc}
0 & 0.1 & 0 & 0.1 & 0 & 0 \\
0 & 0 & 0 & 0 & 0 & 0.1 \\
0 & 0.1 & 0 & 0 & 0.2 & 0 \\
0 & 0.2 & 0 & 0 & 0.3 & 0 \\
0.2 & 0 & 0 & 0 & 0 & 0 \\
0 & 0 & 0.3 & 0 & 0.1 & 0
\end{array}\right), \\
Q^{1}(2) & =\left(\begin{array}{cccccc}
0 & 0 & 0 & 0.2 & 0 & 0 \\
0.1 & 0 & 0.15 & 0 & 0.1 & 0 \\
0 & 0.2 & 0 & 0 & 0 & 0 \\
0 & 0 & 0 & 0 & 0.2 & 0 \\
0 & 0.1 & 0 & 0.15 & 0 & 0.2 \\
0 & 0 & 0.1 & 0 & 0 & 0
\end{array}\right),
\end{aligned}
$$




$$
\begin{aligned}
Q^{2}(1) & =\left(\begin{array}{cccccc}
0 & 0.2 & 0 & 0 & 0 & 0 \\
0 & 0 & 0.1 & 0 & 0 & 0 \\
0.1 & 0 & 0 & 0 & 0 & 0.1 \\
0.1 & 0 & 0 & 0 & 0 & 0.1 \\
0 & 0 & 0 & 0.2 & 0 & 0 \\
0 & 0 & 0 & 0 & 0.1 & 0
\end{array}\right), \\
Q^{2}(2) & =\left(\begin{array}{cccccc}
0 & 0.2 & 0 & 0.2 & 0 & 0 \\
0.2 & 0 & 0 & 0.1 & 0.1 & 0 \\
0 & 0.2 & 0 & 0 & 0 & 0.2 \\
0 & 0 & 0 & 0 & 0.15 & 0 \\
0 & 0 & 0 & 0 & 0 & 0.1 \\
0 & 0 & 0.1 & 0 & 0.15 & 0
\end{array}\right), \\
Q^{3}(1) & =\left(\begin{array}{cccccc}
0 & 0 & 0 & 0 & 0 & 0 \\
0.1 & 0 & 0 & 0 & 0.2 & 0 \\
0 & 0.1 & 0 & 0 & 0 & 0.1 \\
0.1 & 0 & 0 & 0 & 0 & 0 \\
0 & 0.2 & 0 & 0.1 & 0 & 0 \\
0 & 0 & 0 & 0 & 0.1 & 0
\end{array}\right), \\
Q^{3}(2) & =\left(\begin{array}{cccccc}
0 & 0.15 & 0 & 0 & 0 & 0 \\
0 & 0 & 0.1 & 0 & 0 & 0 \\
0 & 0 & 0 & 0 & 0 & 0.2 \\
0.2 & 0 & 0 & 0 & 0 & 0 \\
0 & 0 & 0.2 & 0.1 & 0 & 0 \\
0 & 0 & 0 & 0 & 0.1 & 0
\end{array}\right) .
\end{aligned}
$$

Further, we verify the conditions of Theorem 2 . We can make a direct calculation to get that

$$
\begin{array}{lll}
\sigma_{k}(1)=\frac{-0.6}{k}, & \sigma_{k}(2)=\frac{-1.2}{k}, & \\
\rho_{k}(1)=0.02 k, & \rho_{k}(2)=0.01 k, & k=1,2, \ldots, 6, \\
\eta_{1}(1)=0.5, & \eta_{2}(1)=0.8, & \eta_{3}(1)=1, \\
\eta_{1}(2)=0.8, & \eta_{2}(2)=0.4, & \eta_{3}(2)=0.8 .
\end{array}
$$

Define

$$
D_{k h}(i)=\max _{1 \leq s \leq 3}\left\{\delta_{s}(i) b_{k h}^{s}(i) \eta_{s}(i)\right\}, k, h=1,2, \ldots, 6, i=1,2 .
$$

Then the following two new matrices $D(i)=\left(D_{k h}(i)\right)_{6 \times 6}, i=1,2$ are given:

$$
D(1)=\left(\begin{array}{cccccc}
0 & 0.04 & 0 & 0.04 & 0 & 0 \\
0.02 & 0 & 0.02 & 0 & 0.04 & 0.02 \\
0.02 & 0.02 & 0 & 0 & 0.04 & 0.02 \\
0.02 & 0.04 & 0 & 0 & 0.06 & 0.02 \\
0.04 & 0.04 & 0 & 0.04 & 0 & 0 \\
0 & 0 & 0.06 & 0 & 0.02 & 0
\end{array}\right),
$$




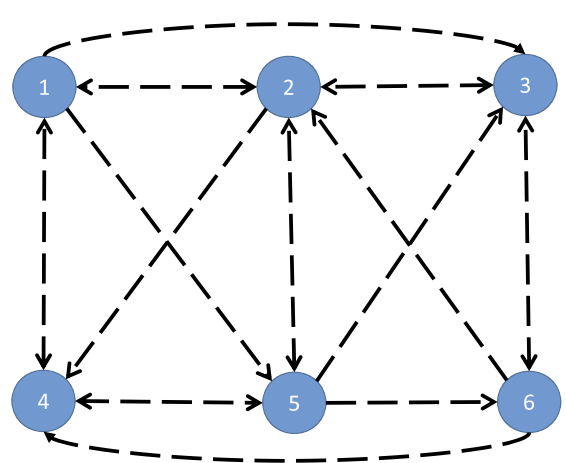

(a) $(\mathcal{G}, D(1))$

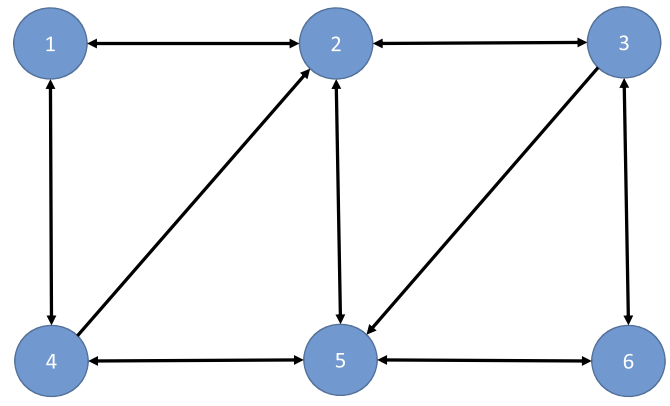

(b) $(\mathcal{G}, D(2))$

Figure 2 The diagram for two diagraphs $(\mathcal{G}, D(1))$ and $(\mathcal{G}, D(2))$

$$
D(2)=\left(\begin{array}{cccccc}
0 & 0.08 & 0 & 0.08 & 0 & 0 \\
0.08 & 0 & 0.06 & 0.04 & 0.04 & 0 \\
0 & 0.08 & 0 & 0 & 0 & 0.08 \\
0.08 & 0 & 0 & 0 & 0.08 & 0 \\
0 & 0.04 & 0.08 & 0.06 & 0 & 0.08 \\
0 & 0 & 0.04 & 0 & 0.06 & 0
\end{array}\right) .
$$

Two new digraphs $(\mathcal{G}, D(1))$ and $(\mathcal{G}, D(2))$ are shown in Fig. 2. Obviously, they are strongly connected.

Let $p=2$. Then the matrices defined by (13) are as follows:

$$
\begin{aligned}
\mathcal{A}_{1}(2) & =-\operatorname{diag}\left(-1.2\left|1+\bar{b}_{1}\right|+0.5,-2.4\left|1+\bar{b}_{1}\right|+0.97\right)-\Gamma \\
& =\left(\begin{array}{cc}
1.2\left|1+\bar{b}_{1}\right|+0.5 & -1 \\
-7 & 2.4\left|1+\bar{b}_{1}\right|+6.03
\end{array}\right), \\
\mathcal{A}_{2}(2) & =-\operatorname{diag}\left(-0.6\left|1+\bar{b}_{2}\right|+0.64,-1.2\left|1+\bar{b}_{2}\right|+1.34\right)-\Gamma \\
& =\left(\begin{array}{cc}
0.6\left|1+\bar{b}_{2}\right|+0.36 & -1 \\
-7 & 1.2\left|1+\bar{b}_{2}\right|+5.66
\end{array}\right), \\
\mathcal{A}_{3}(2) & =-\operatorname{diag}\left(-0.4\left|1+\bar{b}_{3}\right|+0.66,-0.8\left|1+\bar{b}_{3}\right|+0.99\right)-\Gamma \\
& =\left(\begin{array}{cc}
0.4\left|1+\bar{b}_{3}\right|+0.34 & -1 \\
-7 & 0.8\left|1+\bar{b}_{3}\right|+6.01
\end{array}\right),
\end{aligned}
$$




$$
\begin{aligned}
\mathcal{A}_{4}(2) & =-\operatorname{diag}\left(-0.3\left|1+\bar{b}_{4}\right|+0.9,-0.6\left|1+\bar{b}_{4}\right|+1\right)-\Gamma \\
& =\left(\begin{array}{cc}
0.3\left|1+\bar{b}_{4}\right|+0.1 & -1 \\
-7 & 0.6\left|1+\bar{b}_{4}\right|+6
\end{array}\right) \\
\mathcal{A}_{5}(2) & =-\operatorname{diag}\left(-0.24\left|1+\bar{b}_{5}\right|+0.82,-0.48\left|1+\bar{b}_{5}\right|+0.65\right)-\Gamma \\
& =\left(\begin{array}{cc}
0.24\left|1+\bar{b}_{5}\right|+0.18 & -1 \\
-7 & 0.48\left|1+\bar{b}_{5}\right|+6.35
\end{array}\right) \\
\mathcal{A}_{6}(2) & =-\operatorname{diag}\left(-0.2\left|1+\bar{b}_{6}\right|+0.6,-0.4\left|1+\bar{b}_{6}\right|+0.66\right)-\Gamma \\
& =\left(\begin{array}{cc}
0.2\left|1+\bar{b}_{6}\right|+0.4 & -1 \\
-7 & 0.4\left|1+\bar{b}_{6}\right|+6.34
\end{array}\right) .
\end{aligned}
$$

If we choose $\bar{b}_{1} \geq-0.58$ or $\bar{b}_{1} \leq-1.42, \bar{b}_{2} \geq 0.08$ or $\bar{b}_{2} \leq-2.08, \bar{b}_{3} \geq 0.57$ or $\bar{b}_{3} \leq-2.57$, $\bar{b}_{4} \geq 1.73$ or $\bar{b}_{4} \leq-3.73, \bar{b}_{5} \geq 2$ or $\bar{b}_{5} \leq-4, \bar{b}_{6} \geq 1.72$ or $\bar{b}_{6} \leq-3.72$, then $\mathcal{A}_{k}(2)$ is an M-matrix for each $k(k=1,2,3,4,5,6)$. By Theorem 2 and Corollary 1 drive-response networks (16) and (17) are mean square exponentially synchronized and almost surely exponentially synchronized.

Taking the initial data $x_{1}(0)=1.5, x_{2}(0)=-1.2, x_{3}(0)=1.3, x_{4}(0)=-1.4, x_{5}(0)=1.5$, $x_{6}(0)=-1.5, y_{1}(0)=-1.4, y_{2}(0)=1.3, y_{3}(0)=-1.4, y_{4}(0)=1.5, y_{5}(0)=-1.5, y_{6}(0)=1.5$, $\bar{b}_{1}=1, \bar{b}_{2}=2, \bar{b}_{3}=2, \bar{b}_{4}=2.5, \bar{b}_{5}=2.5, \bar{b}_{6}=3$, the simulation results for the second moment and the sample path of drive-response networks (16) and (17) are shown in Fig. 3 and 4, respectively. Figure 3 tells us that drive-response networks (16) and (17) are exponentially synchronized in mean square, whereas Fig. 4 shows almost surely exponential synchronization of (16) and (17). Some sample path of drive-response networks (16) and (17) is presented in Fig. 5, from which we can clearly see that drive-response networks (16) and (17) are synchronized.

Remark 6 In the numerical example, triplet weights and two kinds of noise, white noise and telegraph noise, are considered in complex networks. These factors make it difficult

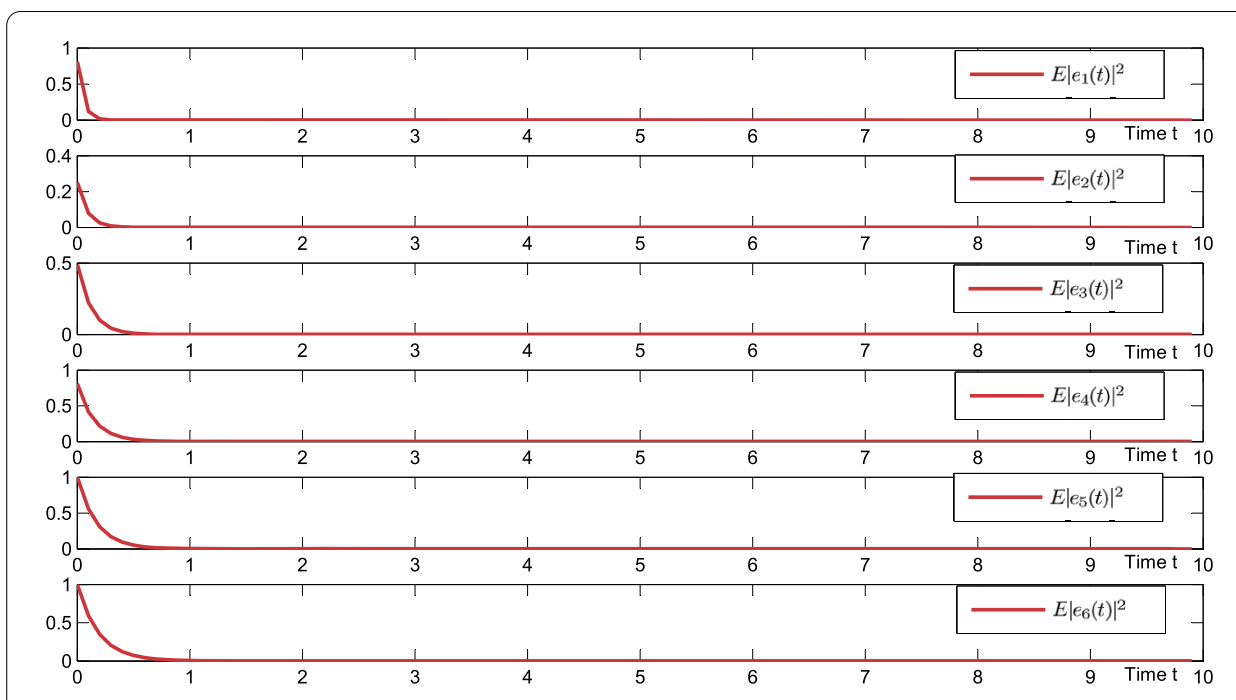

Figure 3 The second moment of solution to synchronization error for drive-response networks (16) and (17) 


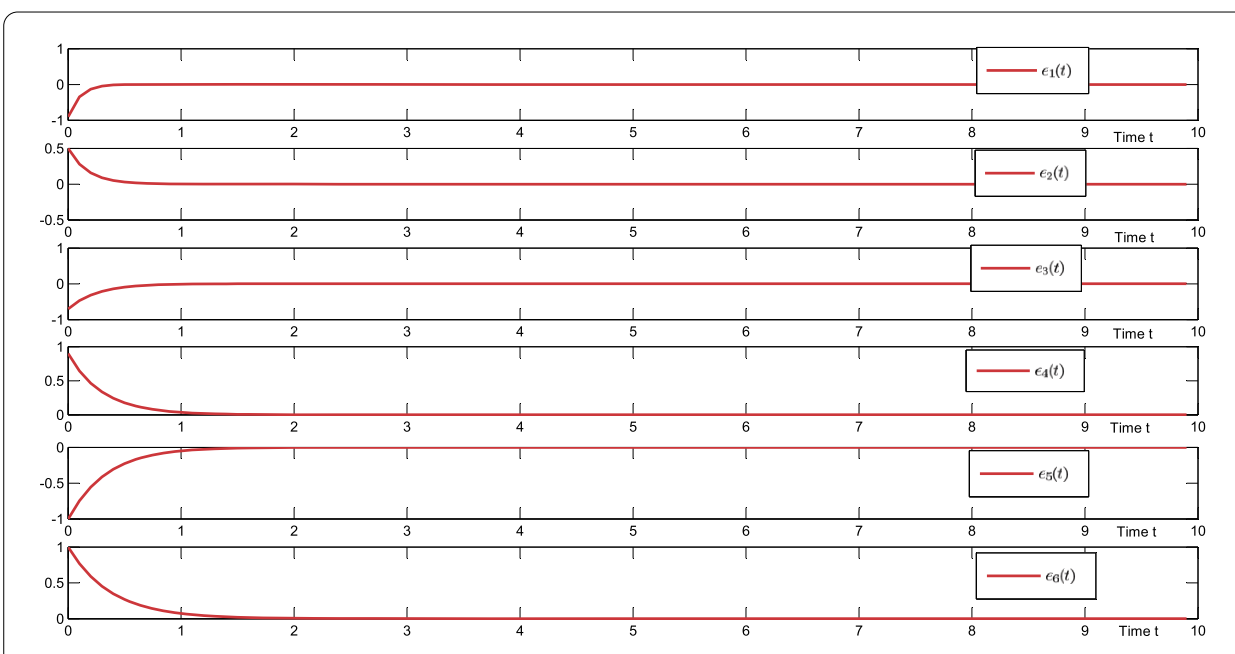

Figure 4 Sample path of synchronization error for drive-response networks (16) and (17)

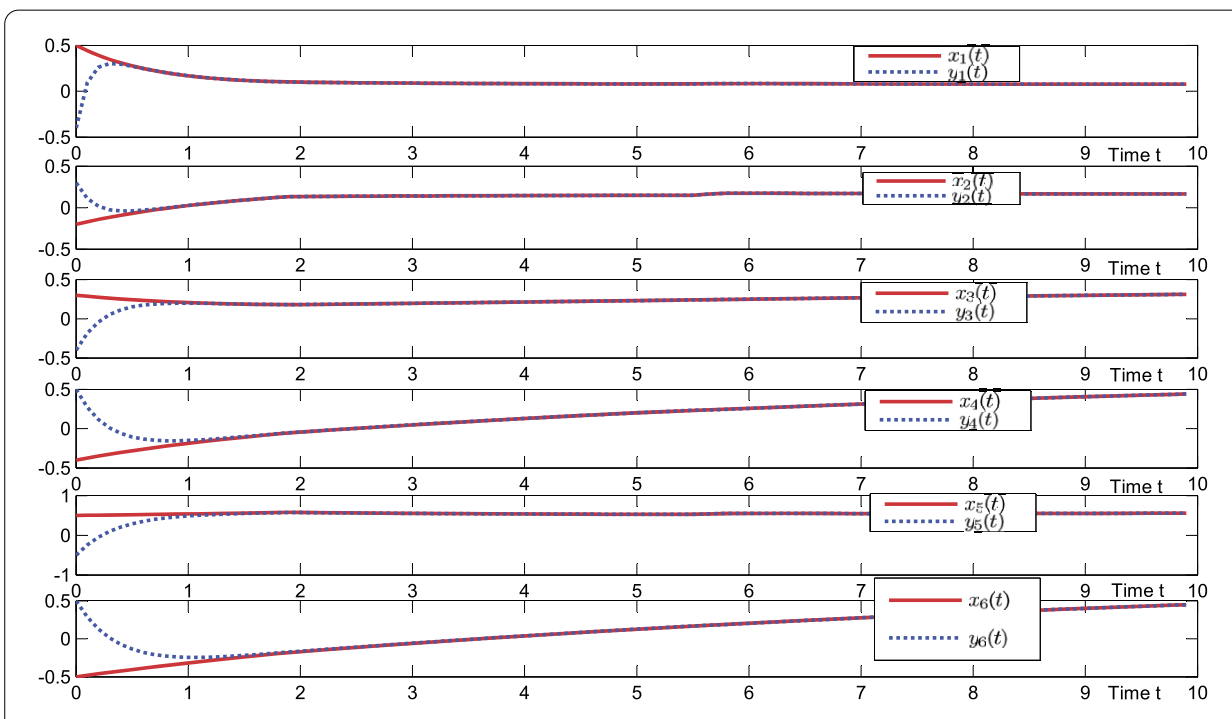

Figure 5 Sample path of drive-response networks (16) and (17)

to achieve the synchronization of drive-response networks. Based on drive-response concept, a novel graph-theoretic method and state feedback control technique are combined to overcome this difficulty. Criteria about $p$ th moment exponential synchronization and almost surely exponential synchronization are provided. Numerical simulations clearly show the effectiveness of theoretical results.

\section{Conclusion}

In this paper, multi-weights and perturbation by white noise and color noise are all considered in the complex networks. Based on the concept of drive-response, the synchronization for stochastic multiple weighted coupled networks with Markovian switching has been investigated. The key point is constructing a global Lyapunov function of coupled networks by using a graph-theoretic method. Then the synchronization criteria have been obtained by combing state feedback control technique and stochastic analysis. Both expo- 
nential synchronization criteria and asymptotical synchronization criteria have been presented. In this paper, drive network and response network have the same topology structure. In real applications, the topology of complex networks is always unknown. So, we can do the topology identification of stochastic multiple weighted networks with Markovian switching based on the synchronization criteria in this paper. This is our future work. Besides, we tend to extend the main results to multi-agent system and cyber-physical system.

\section{Acknowledgements}

The authors would like to thank anonymous reviewers to help us improving the paper.

\section{Funding}

This work was supported by the National Natural Science Foundation of China (No. 11601445) and the Fundamental Research Funds for the Central Universities (No. 2682018CX60).

Availability of data and materials

Not applicable.

\section{Competing interests}

The authors declare that they have no competing interests.

Authors' contributions

All authors contributed equally to this manuscript. All authors read and approved the final manuscript.

\section{Publisher's Note}

Springer Nature remains neutral with regard to jurisdictional claims in published maps and institutional affiliations.

Received: 11 December 2019 Accepted: 6 April 2020 Published online: 16 April 2020

\section{References}

1. Zhang, $X .:$ Wang, J.: Analysis and adaptive control for synchronization and $H_{\infty}$ synchronization of coupled partial differential systems with multiple time-varying delays. J. Franklin Inst. 355, 4351-4378 (2018)

2. Xu, M.: Wang, J.: Wei, P.: Synchronization for coupled reaction-diffusion neural networks with and without multiple time-varying delays via pinning-control. Neurocomputing 227, 82-99 (2017)

3. Guo, B.: Xiao, Y.: Zhang, C.: Synchronization analysis of stochastic coupled systems with time delay on networks by periodically intermittent control and graph-theoretic method. Nonlinear Anal. Hybrid Syst. 30, 118-133 (2018)

4. Ren, S.: Wang, J.: Wu, J.: Generalized passivity of coupled neural networks with directed and undirected topologies. Neurocomputing 314, 371-385 (2018)

5. Dong, H.: Zhou, J.: Wang, B.: Synchronization of nonlinearly and stochastically coupled Markovian switching networks via event-triggered sampling. IEEE Trans. Neural Netw. Learn. Syst. 99, 1-10 (2018)

6. Tan, M.: Li, X.: Liu, Y.: Finite-time stability and synchronization of the coupled switched neural networks with nodes of different dimensions. Neural Process. Lett. 49, 285-303 (2019)

7. Sakthivel, R.: Sakthivel, R.: Kaviarasan, B.: Finite-time nonfragile synchronization of stochastic complex dynamical networks with semi-Markov switching outer coupling. Complexity 2018, Article ID 13 (2018)

8. Sakthivel, R.: Sathishkumar, M.: Kaviarasan, B.: Synchronization and state estimation for stochastic complex networks with uncertain inner coupling. Neurocomputing 238, 44-55 (2017)

9. Chen, Y.: Yu, W.: Tan, S.: Synchronizing nonlinear complex networks via switching disconnected topology. Automatica 70, 189-194 (2016)

10. Xu, C.: Su, H.: Liu, C.: Robust adaptive synchronization of complex network with bounded disturbances. Adv. Differ. Equ. 2019, Article ID 483 (2019)

11. Li, X.: Yang, G.: Graph theory-based pinning synchronization of stochastic complex dynamical networks. IEEE Trans. Neural Netw. Learn. Syst. 28, 427-437 (2017)

12. Shi, L.: Zhu, H.: Synchronization for time-varying complex networks based on control. J. Comput. Appl. Math. 301, 178-187 (2016)

13. Feng, Y.: Xiong, X.: Exponential synchronization of inertial neural networks with mixed delays via quantized pinning control. Neurocomputing 310, 165-171 (2018)

14. Kaviarasan, B.: Kwon, O.M.: Park, M.J.: Composite synchronization control for delayed coupling complex dynamical networks via a disturbance observer-based method. Nonlinear Dyn. 99, 1601-1619 (2020)

15. Zhang, D.: Shen, Y.: Mei, J.: Finite-time synchronization of multi-layer nonlinear coupled complex networks via intermittent feedback control. Neurocomputing 225, 129-138 (2017)

16. Li, F.: Li, J.: State feedback controller design for the synchronization of Boolean networks with time delays. Physica A 490, 1267-1269 (2018)

17. Li, Y.: Wang, H.: Almost periodic synchronization of quaternion-valued shunting inhibitory cellular neural networks with mixed delays via state-feedback control. PLoS ONE 13, Article ID e0198297 (2018)

18. Wu, Y.: Chen, B.: Li, W.: Synchronization of stochastic coupled systems via feedback control based on discrete-time state observations. Nonlinear Anal. Hybrid Syst. 26, 68-85 (2017) 
19. Majhi, S.: Bera, B.: Banerjee, S.: Synchronization of chaotic modulated time delay networks in presence of noise. Eur. Phys. J. Spec. Top. 225, 65-74 (2016)

20. Ray, A.: Chowdhury, A.R.: Ghosh, D.: Effect of noise on chaos synchronization in time-delayed systems: numerical and experimental observations. Physica A 392, 4837-4849 (2013)

21. Zhu, Q: Stabilization of stochastic nonlinear delay systems with exogenous disturbances and the event-triggered feedback control. IEEE Trans. Autom. Control 64, 3764-3771 (2019)

22. Zhang, C.: Shi, L.: Exponential synchronization of stochastic complex networks with multi-weights: a graph-theoretic approach. J. Franklin Inst. 356, 4106-4123 (2019)

23. Pan, L.: Cao, J.: Hu, J.: Synchronization for complex networks with Markov switching via matrix measure approach. Appl. Math. Model. 39, 5636-5649 (2015)

24. Wang, X.: Yang, B.: Gao, K.: Finite-time synchronization control relationship analysis of two classes of Markovian switched complex networks. Int. J. Control. Autom. Syst. 16, 2845-2858 (2018)

25. Sheng, S.: Zhang, X.: Lu, G.: Finite-time outer-synchronization for complex networks with Markov jump topology via hybrid control and its application to image encryption. J. Franklin Inst. 355, 6493-6519 (2018)

26. Zhang, C.: Li, W.: Wang, K.: Graph-theoretic method on exponential synchronization of stochastic coupled networks with Markovian switching. Nonlinear Anal. Hybrid Syst. 15, 37-51 (2015)

27. Ren, H.: Deng, F.: Peng, Y.: Finite time synchronization of Markovian jumping stochastic complex dynamical systems with mix delays via hybrid control strategy. Neurocomputing 272, 683-693 (2018)

28. Zhao, H.: Li, L.: Peng, H.: Finite-time topology identification and stochastic synchronization of complex network with multiple time delays. Neurocomputing 219, 39-49 (2017)

29. Zhang, C.: Han, B.: Stability analysis of stochastic delayed complex networks with multi-weights based on Razumikhin technique and graph theory. Physica A 538, Article ID 122827 (2020)

30. Kaviarasan, B.: Kwon, O.-M.: Park, M.J.: Integrated synchronization and anti-disturbance control design for fuzzy model-based multiweighted complex network. IEEE Trans. Syst. Man Cybern. Syst. (2020). https://doi.org/10.1109/TSMC.2019.2960803

31. Qiu, S.: Huang, Y.: Ren, S.: Finite-time synchronization of multi-weighted complex dynamical networks with and without coupling delay. Neurocomputing 275, 1250-1260 (2018)

32. An, X.: Zhang, L.: Li, Y: Synchronization analysis of complex networks with multi-weights and its application in public traffic network. Physica A 412, 149-156 (2014)

33. Qin, Z.: Wang, J.: Synchronization and $H_{\infty}$ synchronization of multi-weighted complex delayed dynamical networks with fixed and switching topologies. J. Franklin Inst. 354, 7119-7138 (2017)

34. Du, W.: Li, Y.: Zhang, J.: Synchronisation between two different networks with multi-weights and its application in public traffic network. Int. J. Syst. Sci. 50, 534-545 (2019)

35. Zhou, H.: Zhang, Y:: Li, W.: Synchronization of stochastic Lévy noise systems on a multi-weights network and its applications of Chua's circuits. IEEE Trans. Circuits Syst. I, Regul. Pap. 66, 2709-2722 (2019)

36. Wang, Q.: Wang, J.: Huang, Y:: Generalized lag synchronization of multiple weighted complex networks with and without time delay. J. Franklin Inst. 355, 6597-6616 (2018)

37. Li, M.Y.: Shuai, Z:: Global-stability problem for coupled systems of differential equations on networks. J. Differ. Equ. $248,1-20(2010)$

38. Liu, Y.: Yu, P.: Chu, D.: Stationary distribution of stochastic multi-group models with dispersal and telegraph noise. Nonlinear Anal. Hybrid Syst. 33, 93-103 (2019)

39. Zhang, C.: Deng, F: Mao, W. Synchronization of complex stochastic coupled systems based on graph theory. IEEE Access 6, 68867-68873 (2018)

40. Wu, Y.: Fu, S.: Li, W.: Exponential synchronization for coupled complex networks with time-varying delays and stochastic perturbations via impulsive control. J. Franklin Inst. 356, 492-513 (2019)

41. Wu, Y: Wang, C.: Li, W: Generalized quantized intermittent control with adaptive strategy on finite-time synchronization of delayed coupled systems and applications. Nonlinear Dyn. 95, 1361-1377 (2019)

42. Wang, P.: Feng, J.: Su, H.: Stabilization of stochastic delayed networks with Markovian switching and hybrid nonlinear coupling via aperiodically intermittent control. Nonlinear Anal. Hybrid Syst. 32, 115-130 (2019)

43. Zhang, C.: Chen, T.: Exponential stability of stochastic complex networks with multi-weights based on graph theory. Physica A 496, 602-611 (2018)

44. Mao, X:: Stochastic Differential Equations with Markovian Switching. Imperial College Press, London (2006)

45. West, D.B.: Introduction to Graph Theory. Prentice Hall, Upper Saddle River (1996)

46. Zhang, C.: Yang, Y: Synchronization of stochastic multi-weighted complex networks with Lévy noise based on graph theory. Physica A 545, Article ID 123496 (2020)

\section{Submit your manuscript to a SpringerOpen ${ }^{\mathcal{O}}$ journal and benefit from:}

- Convenient online submission

- Rigorous peer review

- Open access: articles freely available online

- High visibility within the field

Retaining the copyright to your article

Submit your next manuscript at $\gg$ springeropen.com 